\title{
A national survey of Ixodidae ticks on privately owned dogs in Italy
}

\author{
Maria Paola Maurelli', Paola Pepe ${ }^{1}$, Liliana Colombo ${ }^{2}$, Rob Armstrong $^{3 *}$ (D), Elena Battisti ${ }^{4}$, Maria Elena Morgoglione ${ }^{1}$, \\ Dimitris Counturis ${ }^{4}$, Laura Rinaldii', Giuseppe Cringoli ${ }^{1}$, Ezio Ferroglio ${ }^{4}$ and Stefania Zanet ${ }^{4}$
}

\begin{abstract}
Background: The geographical distribution of ticks on companion animals needs to be monitored to develop and plan effective control measures, as suggested by the European Scientific Counsel on Companion Animal Parasites. The aim of this study was to conduct the first Italian national survey of tick distribution on privately owned dogs.

Methods: The study was performed over 20 months (February 2016 - September 2017) and involved 153 veterinary practices in 64 different provinces covering 17/20 (85\%) Italian regions. Participating practitioners were asked to examine five different dogs per month at random and complete a questionnaire for each dog. Differences in tick infestation associated with: sex, age and hair length (long and short); the dog's habitat (indoor or outdoor/kennel); and the dog's environment (urban or rural/sylvatic) were evaluated. The attachment site of ticks on the dog was also recorded. Acaricide efficacy was evaluated for the subset of dogs for which complete information on product used, date of sampling and date of last ectoparasiticide treatment was available.
\end{abstract}

Results: Of the 3026 dogs examined, 1383 (45.7\%) were carrying at least one tick. Overall, 2439 tick samples were collected and a total of 14 tick species identified. Rhipicephalus sanguineus group were the most predominant ticks (63.6\%), followed by lxodes ricinus (30.6\%) and I. hexagonus (5.6\%). Twenty-four dogs had mixed tick infestations. Long-haired dogs had a higher tick infestation risk as did dogs with outdoor and rural/sylvatic lifestyles. Ticks were located on the head (37.4\%), the neck (28.8\%), the muzzle (15.5\%) and the back (15.3\%). A higher prevalence of Rhipicephalus was found in the interdigital spaces (10.8\%) compared to Ixodes (0.2\%). Finally, ectoparasiticide treatments were found significantly protective against tick infestation, especially orally administered formulations.

Conclusions: Privately owned dogs in Italy have a high prevalence (45.7\%) of infestation with ixodid ticks and this risk varies by dog phenotype and lifestyle.

Keywords: Ticks, Dogs, Epidemiology, Risk factors, Seasonal distribution, Italy

\section{Background}

In Animal Planet's list of the top ten most extreme bloodsuckers on Earth, mosquitoes were sixth, leeches fifth, kissing bugs fourth, bedbugs third, fleas second and ticks first $[1,2]$. The literature reports either mosquitoes or ticks as the most important vectors of pathogens to animals and humans [2]. Hard ticks (Ixodidae) are ectoparasites of domestic and wild animals, as well as humans. Their medical and veterinary importance is increased by their great capacity for transmitting viral, bacterial, protozoan and helminthic infections to animals, causing a diverse range of

\footnotetext{
* Correspondence: robert.armstrong@merck.com

${ }^{3}$ MSD Animal Health, Madison, NJ, USA

Full list of author information is available at the end of the article
}

infections commonly referred to as tick-borne diseases (TBDs) [3]. Although chemical tick control options are available, the worldwide incidence of human TBDs is increasing. In Europe, an estimated average of 85,000 people are diagnosed every year with Lyme borreliosis [3]. The incidence of human TBDs increased in Italy over the last decade, with 4604 clinical cases and 33 deaths documented by the Ministry of Health in the period 1998-2002, mainly in southern and insular regions [3].

Tick geographical distribution and abundance are influenced by biotic and abiotic factors, such as climate, altitude, urbanization and host population dynamics [4]. In recent years, environmental changes, and the movement of people and animals have introduced novel vector species into

(c) The Author(s). 2018 Open Access This article is distributed under the terms of the Creative Commons Attribution 4.0 International License (http://creativecommons.org/licenses/by/4.0/), which permits unrestricted use, distribution, and 
previously free areas, leading to changes in local epidemiology of ticks and their associated pathogens $[5,6]$. Reducing and controlling these ectoparasites is extremely difficult. Currently, control measures are mainly based on use of chemicals on animals and in the environment [7]. For the multiple reasons mentioned above, it is crucial to develop and implement a systematic surveillance system, based on a comprehensive knowledge of tick species present in a target geographical area [8, 9]. Companion animals, especially dogs, may be useful sentinels for monitoring tick population distribution and also the pathogens they carry $[10,11]$. Many national surveys have been conducted in Europe, based on this assumption, to measure tick abundance on dogs and to understand their spatial distribution $[9,10,12-14]$. Italy is a European country with many species of ticks, with about 40 different species reported [3]. However, to date, there has been no surveillance program in Italy, and information regarding Italian ixodofauna of dogs is limited to local surveys of single tick species and their pathogens [15], or to ticks collected from the environment [16-20].

The aim of this study was to conduct the first national Italian survey of ticks in privately owned dogs presented to veterinary practices and to develop a spatial distribution framework of different tick species. Risk factors associated with tick infestation, seasonality and acaricide efficacy were recorded and analyzed.

\section{Methods}

\section{Study design and tick collection}

A nationwide survey was performed in Italy from February 2016 to September 2017. The project involved voluntary participation of veterinary practices in different areas of Italy. The dogs enrolled in the study were homogeneously distributed across the regions using a criterion of proportional allocation, i.e. each region was assigned a number of dogs proportional to the total number of dogs registered in each region [21]. Each veterinary practice was provided with a "tick survey package" containing the study protocol, 60 questionnaires, 60 sample vials with $70 \%$ ethanol and a tick removal hook. The protocol instructed participating vets to examine at least five dogs per month for ticks over the 20-month study period. Dogs were to be randomly chosen without prior knowledge of their tick infestation status. The questionnaire requested information on the location of the sampled dog (post code of the owner or, if this was not given, the practice postcode), breed, sex, age, hair length and recent ectoparasiticidal treatment (the last treatment for ticks and the drug used). Additional questions included: the type of housing (indoor or outdoor/kennel), the environment in which dog is usually moved (urban, rural/sylvatic), and the attachment site of ticks if present.
At the time of the visit, each dog was thoroughly visually examined for 15-20 min to detect any ticks present. Eleven body regions were observed including: head, muzzle, neck, armpits, back, abdomen, arts, tail, anus, vagina and interdigital spaces. All ticks found were removed and preserved in the sample vials at room temperature for submission to the laboratories of Parasitology in Turin and Naples. An individual identification number (ID) was assigned to each animal and questionnaires and vials were labeled with the same ID.

\section{Data handling and tick identification}

Questionnaire and sample vial data were entered into an Excel (Microsoft, Redmond, WA, USA) spreadsheet. Ticks were identified to species level, life-stage (i.e. larva, nymph or adult) and gender (female or male) under a stereomicroscope using appropriate morphological keys [22-24].

Specimens (86) that were difficult to identify from morphological characterization were selected for genetic analysis. DNA was extracted using a commercial kit (HiPurA $^{\mathrm{Tm}}$ PCR Product Purification Kit, HiMedia, Mumbai, India) in accordance with the manufacturer's instructions. Partial mitochondrial $12 S$ rRNA and $16 S$ rRNA gene sequences were generated and analyzed using primers and PCR conditions as previously described [24, 25]. Amplicons were resolved in ethidium bromide-stained (1.5\%) agarose gels (Bio-Rad, Madrid, Spain) and sized by comparison with a marker in the $6 \mathrm{X}$ DNA Loading Dye (Thermo Fisher Scientific, Waltham, USA). Gels were photographed using a digital documentation system (Gel Doc 2000, Bio-Rad, Watford, UK). PCR products (amplicons) were purified (HiPurA ${ }^{\mathrm{TM}}$ Mammalian Genomic DNA Purification Kit, HiMedia) and sequenced. Sequences were analyzed using the Chromas version 2.1.1 software and compared with the partial mitochondrial $12 S$ and $16 S$ rRNA gene sequences in GenBank.

\section{Mapping and statistical analysis}

The location of positive dogs for each tick genera was geo-referenced using a geographical information system (GIS, ArcGIS version 10.3 ESRI), referring to the owner's postcode or, if missing, to the veterinary practice postcode for the dog. GIS was also used to analyze spatial information on body locations of ticks on examined dogs.

Tick infestation differences were analyzed in association with dog sex, age, hair length (long and short), habitat (indoor or outdoor/kennel) and environment (urban or rural/ sylvatic) using the Chi-square test, with presence or absence of ticks as dependent variable. Four categories were used for dog gender: male, female, male neutered and female neutered. Dogs were classed into five groups for the age analysis: puppies (less than 1 year of age); young-adult (1-3 
years); adult (4-6 years); old (7-10 years); and very old ( $>10$ years). Acaricide efficacy was evaluated for those dogs for which complete information on the product used, date of sampling and date of last acaricide treatment was available. Differences were considered significant at $P<0.05$. Chi-square tests and logistic regression were performed using SPSS Statistics 20.0 (IBM Corp. Released 2011. IBM SPSS Statistics for Windows, Version 20.0. Armonk, NY, USA).

\section{Results}

A total of 153 veterinary practices from 64 different provinces of 17/20 (85.0\%) regions of Italy (Fig. 1) participated in the survey and 3026 dogs from northern (1520), central (283) and southern (1223) Italy were examined and had a completed questionnaire. The enrolled dogs included: 835 intact $(27.6 \%$; 95\% CI: 26.0-29.2\%) and 562 neutered females (18.6\%; 95\% CI: $17.2-20.0 \%), 1430$ intact (47.3\%; 95\% CI: $45.5-49.1 \%)$ and 199 neutered males (6.6\%; 95\% CI: 5.7-7.5\%). The age of animals ranged from 1 month to 17 years (median age of 4 years). The dogs belonged to over 100 different breeds, of which cross-bred were the most prevalent (39.3\%; 95\% CI: 37.5-41.0\%). Most had short hair (56.6\%; 95\% CI: 54.9-58.4\%) and were housed rather than kenneled (57.2\%; 95\% CI: 55.5-59.0\%).
Of these, 1383 dogs (45.7\%, 95\% CI: 43.9-47.5\%) were carrying at least one tick; however, ticks were submitted for only 1217 dogs. The body surface was divided in 11 areas and ticks were located on the head (37.4\%), the neck (28.8\%), the muzzle (15.5\%) and the back (15.3\%) (Fig. 2a). No significant differences were found either between the overall tick preference for location of attachment on dogs or between the different tick genera regarding their location of preference on the dog's body. However, a higher prevalence of Rhipicephalus spp. was found in interdigital spaces (10.8\%) compared to Ixodes spp. (0.2\%) (Fig. 2b).

A total of 2439 tick specimens were collected. The median number of ticks per dog was 1 , although the number of ticks per animal ranged between 1-44. Specifically, 58 (2.4\%) larvae, 325 (13.3\%) nymphs and 2056 (84.3\%) adults (1373 females and 683 males), belonging to four genera and 14 species (Fig. 3 and Table 1), were found. Rhipicephalus and Ixodes were the most prevalent genera on dogs in Italy, with Rhipicephalus spp. on $27.5 \%$ of dogs in the north and $36.1 \%$ of dogs in the central-southern regions while Ixodes spp. were found on $25.6 \%$ of northern dogs and $10.8 \%$ of central-southern region dogs. Very few dogs were infested with Dermacentor spp. (0.6\%) or Haemaphysalis spp. (0.2\%) ticks. Rhipicephalus sanguineus group was most predominant (63.6\%), followed by Ixodes

\section{N. Enrolled dogs}
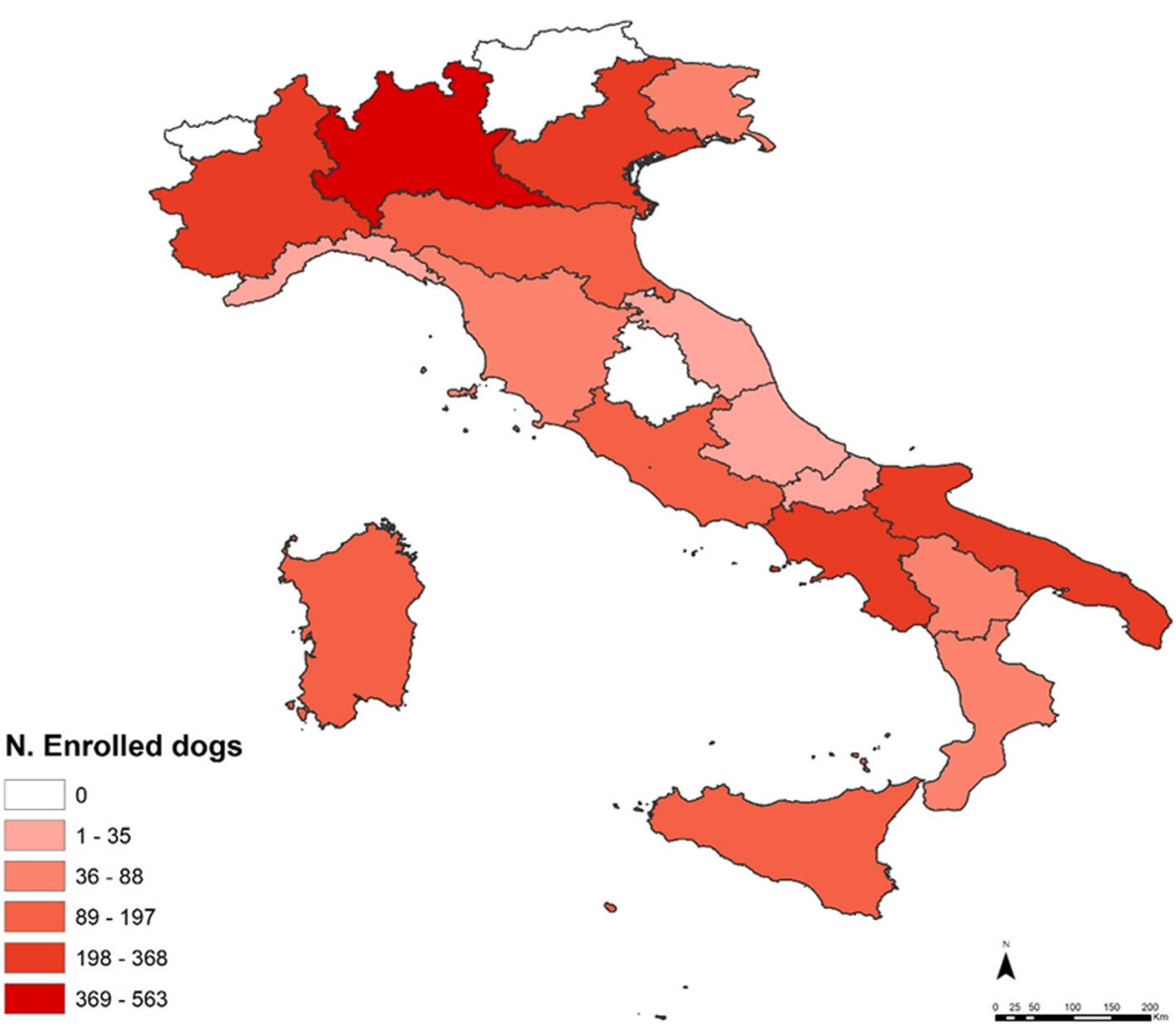

Fig. 1 Dogs enrolled in a survey of tick infestation in Italy showing the frequency of dog enrollment for each Italian region 


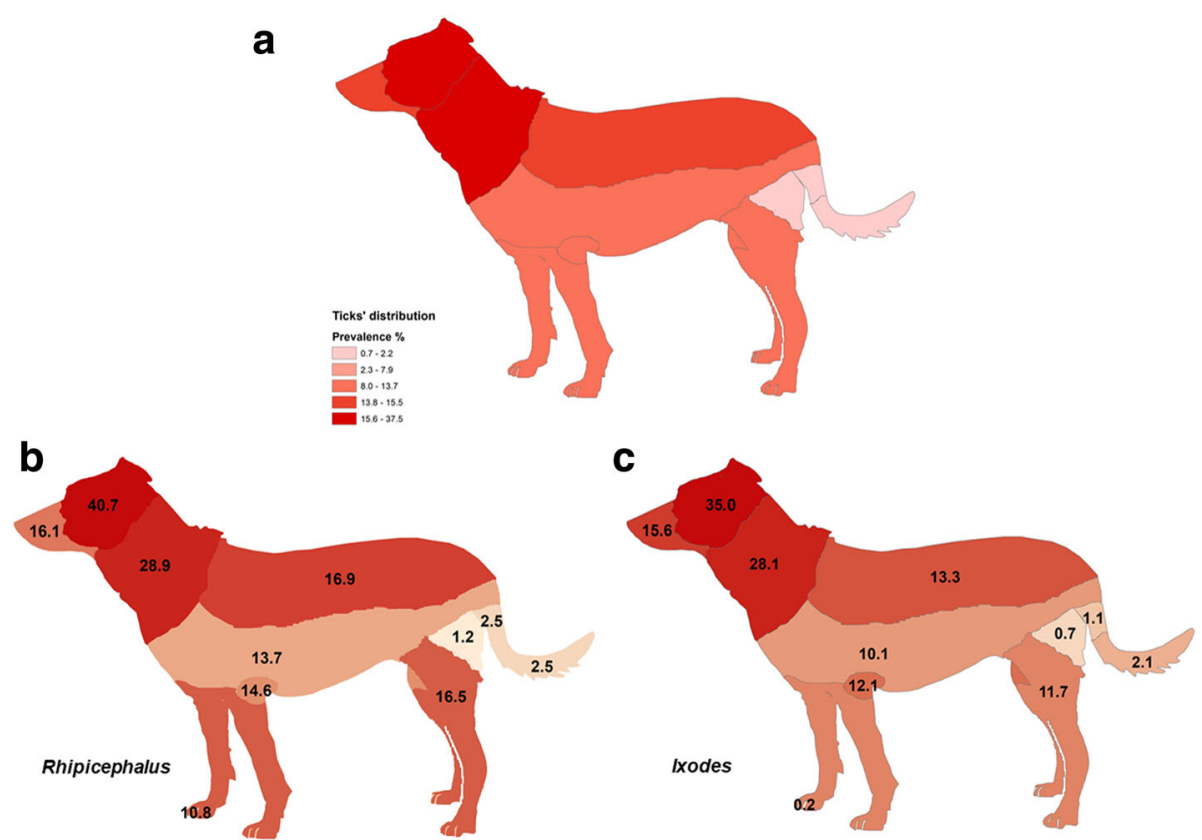

Fig. 2 Distribution of ticks on the bodies of dogs enrolled in an Italian survey: a total ticks collected; b Rhipicephalus spp.; and c /xodes spp. (data elaborated with ArCGIS 10.3)
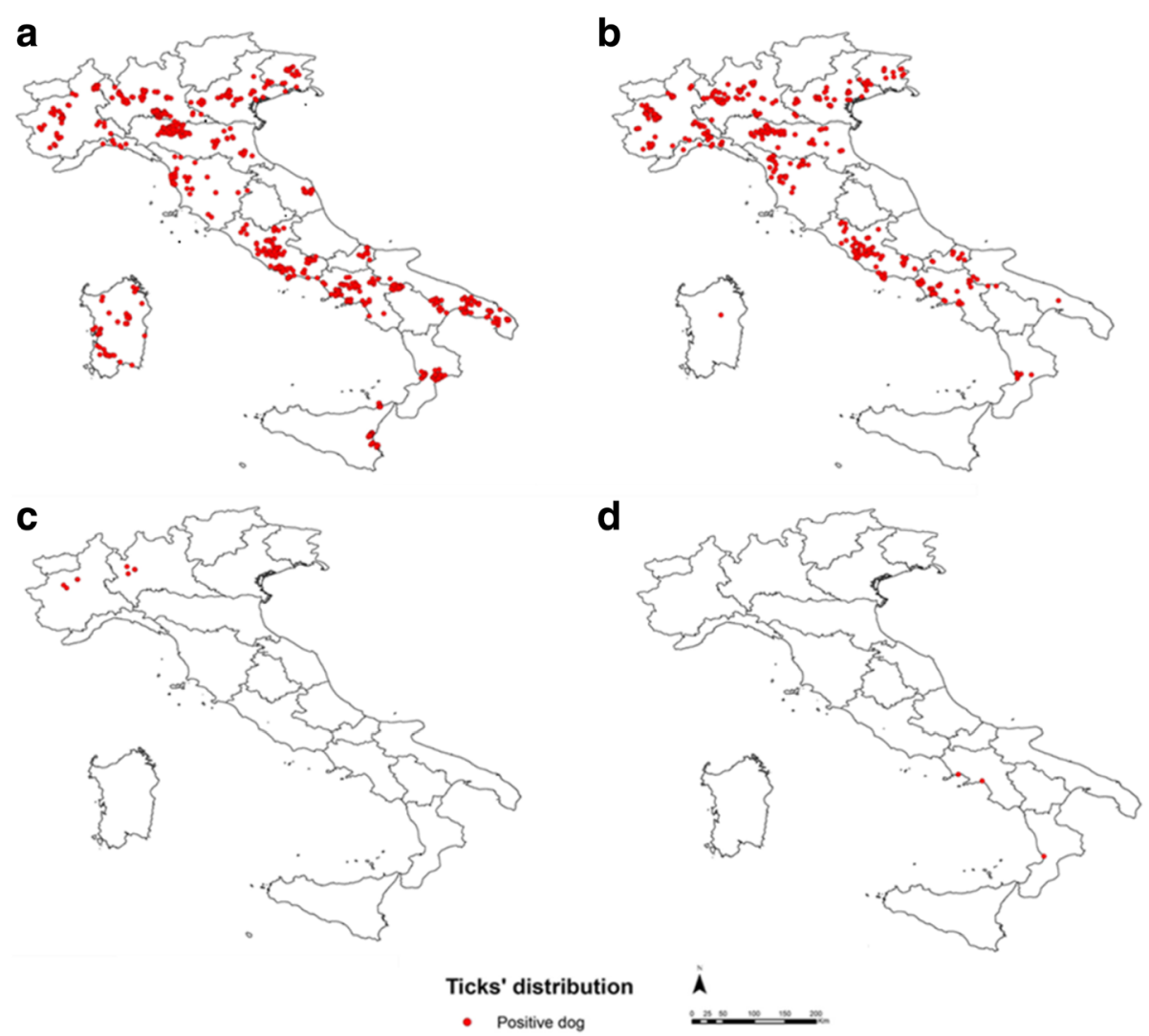

Fig. 3 Point distribution maps of four tick genera: a Rhipicephalus; b /xodes; c Dermacentor; and $\mathbf{d}$ Haemaphysalis, identified in owned dogs surveyed in Italy (2016-2017) (data elaborated with ArcGIS 10.3) 
Table 1 Tick species collected from privately owned dogs in Italy classified by life-stage and sex (larvae, nymphs, females and males)

\begin{tabular}{|c|c|c|c|c|c|}
\hline \multirow[t]{2}{*}{ Tick group/species } & \multirow[t]{2}{*}{ Larvae } & \multirow[t]{2}{*}{ Nymphs } & \multicolumn{2}{|l|}{ Adults } & \multirow{2}{*}{$\begin{array}{l}\text { Total no. } \\
\text { of } \\
\text { specimens }\end{array}$} \\
\hline & & & Females & Males & \\
\hline Rhipicephalus sanguineus group ${ }^{a}$ & 21 & 295 & 881 & 625 & 1822 \\
\hline Ixodes ricinus & 5 & 18 & 399 & 46 & 468 \\
\hline Ixodes hexagonus & 0 & 7 & 73 & 3 & 83 \\
\hline Dermacentor marginatus & 0 & 0 & 4 & 1 & 5 \\
\hline Rhipicephalus bursa & 0 & 1 & 7 & 3 & 11 \\
\hline Dermacentor reticulatus & 0 & 0 & 2 & 5 & 7 \\
\hline Haemaphysalis punctata & 0 & 2 & 2 & 0 & 4 \\
\hline Ixodes arboricola & 32 & 0 & 0 & 0 & 32 \\
\hline Ixodes canisuga & 0 & 2 & 2 & 0 & 4 \\
\hline Ixodes gibbosus & 0 & 0 & 2 & 0 & 2 \\
\hline Ixodes festai & 0 & 0 & 1 & 0 & 1 \\
\hline Total & 58 & 325 & 1373 & 683 & 2439 \\
\hline
\end{tabular}

${ }^{a}$ This group includes Rhipicephalus sanguineus (sensu lato), R. pusillus, R. turanicus and Rhipicephalus sp. I specimens (data confirmed by molecular analysis)

ricinus (30.6\%) and I. hexagonus (5.6\%) (Table 2). A total of 14 species of ticks were identified showing a different pattern of distribution across Italy (Table 3). Mixed infestations with more than one tick species were recorded on 24 dogs (Table 4).

A clear sequence was obtained for only 80 specimens and then compared with GenBank sequences, namely 37 partial $12 S$ rRNA and 43 partial $16 S$ rRNA. The length of the $12 S$ rRNA and $16 S$ rRNA gene sequences alignments were of 370 and $330 \mathrm{bp}$, respectively. This sequence analysis showed 99-100\% identity to GenBank sequences of Rhipicephalus sanguineus (sensu lato) (accession numbers: KU255852, KU255849, KU255848, KU556694, KX553960), $R$. turanicus (accession number: KC243822) and Rhipicephalus sp. I (accession number: KC243794). Additionally, D. marginatus (accession number: JX051098) and D. reticulatus (accession number: JF928493) were 99-100\% identity to GenBank sequences. Finally, five partial $16 S$ rRNA gene sequences were identified as I. canisuga (accession number: KY962075) and I. festai (accession number: KU170522).

\section{Statistical analysis}

The Chi-square test showed that hair length was significantly associated with tick presence $\left(\chi^{2}=5.07, d f=\right.$ $1, P=0.024)$. Dog activity and habitat also influenced tick infestation with outdoor $\left(\chi^{2}=175.3, d f=1, P<0.0001\right)$ and $\mathrm{rural} / \mathrm{sylvatic}\left(\chi^{2}=287.1, d f=1, P<0.0001\right) \mathrm{dogs}$ showing higher prevalence.

\section{Seasonality}

Most samples were received between May 2016 and July 2017, during different seasons (Table 3), with fewer submissions at the beginning and end of the survey period, likely associated with reduced practitioner compliance.

Table 2 Prevalence of tick infestation on dogs surveyed in Italy classified by tick species

\begin{tabular}{llll}
\hline Tick species & Number of dogs & Prevalence (\%) & \\
\hline Rhipicephalus sanguineus group & 769 & 63.6 & $60.40-65.89$ \\
Ixodes ricinus & 372 & 30.6 & $28.00-33.26$ \\
Ixodes hexagonus & 68 & 5.6 & $4.39-7.07$ \\
Rhipicephalus. bursa & 7 & 0.58 & $0.25-1.24$ \\
Dermacentor marginatus & 5 & 0.41 & $0.15-1.02$ \\
Dermaceontor reticulatus & 3 & 0.25 & $0.06-0.78$ \\
Haemaphysalis punctata & 3 & 0.25 & $0.06-0.78$ \\
Ixodes arboricola & 2 & 0.16 & $0.03-0.66$ \\
Ixodes canisuga & 2 & 0.16 & $0.03-0.66$ \\
Ixodes gibbosus & 2 & 0.16 & $0.03-0.66$ \\
Ixodes festai & 1 & 0.08 & $0.00-0.53$ \\
\hline
\end{tabular}

${ }^{\mathrm{a}}$ Total number of dogs on which ticks were collected $=1217$ 
Table 3 Ixodid species identified in a survey of dogs at veterinary practices in Italy by geographical origin and collection season

\begin{tabular}{lll}
\hline Tick species & Geographical origin & Season \\
\hline Dermacentor marginatus & North (Lombardy, Piedmont) & Spring, Summer \\
Dermacentor reticulatus & North (Lombardy, Piedmont) & Spring, Summer \\
Haemaphysalis punctata & South (Calabria, Campania) & Summer, Winter \\
Ixodes arboricola & North (Veneto) & Summer \\
Ixodes canisuga & North (Lombardy) & Summer \\
Ixodes festai & North (Piedmont) & Summer \\
Ixodes gibbosus & South (Calabria, Campania) & Spring \\
Ixodes hexagonus & North (Emilia Romagna, Friuli Venezia Giulia, Lombardy, & Spring, Summer, Autumn, Winter \\
& Piedmont, Veneto), Center (Lazio, Molise, Tuscany), South & \\
Ixodes ricinus & (Calabria, Campania) & Spring, Summer, Autumn, Winter \\
& North (Emilia Romagna, Friuli Venezia Giulia, Lombardy, & \\
Rhipicephalus sp. I & Piedmont, Veneto), Center (Lazio, Molise, Tuscany), & Spring, Summer \\
Rhipicephalus bursa & South (Basilicata, Calabria, Campania, Apulia, Sardinia) & Summer, Winter \\
Rhipicephalus pusillus & South (Basilicata, Apulia) & Spring, Summer, Autumn, Winter \\
Rhipicephalus sanguineus (sensu lato) & Center (Lazio), South (Calabria, Campania, Sicily) & Spring, Summer, Autumn, Winter \\
Rhipicephalus. turanicus & Center (Lazio), South (Basilicata, Calabria, Campania, Apulia) & Spring, Summer, Autumn, Winter
\end{tabular}

The sample collection was homogeneous in different regions throughout the study period.

More dogs were tick-infested during April to August and the number of tick-infested dogs declined from October to February, although Rhipicephalus and Ixodes ticks were collected during all months of the year. Ticks of the Rhipicephalus sanguineus group showed a peak of infestation during spring and summer while I. ricinus and I. hexagonus had a lower variation throughout the year (Fig. 4).

\section{Acaricide treatments}

Acaricide treatments were conducted on 2180 dogs (72.0\%) in the study, although information on the ectoparasiticide product used for treatment was reported only for 2016 dogs. A total of 687 dogs were not treated with any ectoparasiticide product, while for 159 dogs no information was reported regarding ectoparasiticide use. The majority of treated dogs (1930/2016 dogs; 95.7\%) were treated with one product, most frequently a topical spot-on formulation (1278/1930 dogs; 66.2\%) followed by oral formulations (348/1930 dogs; $18.0 \%)$ and collars (283/1930 dogs; 14.7\%). Other formulation types (injectable, spray, shampoo and powder) were each used by $<1 \%$ of the screened dogs. Eighty-six dogs were reported to have been treated with a combination of two products. Spot-ons and collars was the most common association, used on 38 dogs.

The ectoparasiticide treatment efficacy was evaluated by comparing tick infestation in untreated dogs to dogs for which information on the product used and the last treatment date was available: (687 untreated dogs and 1320 dogs with complete ectoparasiticide information). Generally, a history of ectoparasiticide treatment was significantly protective against tick infestation $\left(\chi^{2}=\right.$ 196.89, $d f=1, P<0.0001)$. Differences in the tick control efficacies of oral, spot-on and collar formulations were significant. Considering the recommended retreatment

Table 4 Dogs in a tick survey in Italy infested with a mixed tick infestations

\begin{tabular}{lc}
\hline Tick species & Number of dogs \\
\hline Rhipicephalus sanguineus group + Ixodes ricinus & 10 \\
Rhipicephalus sanguineus group + Ixodes hexagonus & 5 \\
Ixodes ricinus + Ixodes hexagonus & 5 \\
Rhipicephalus sanguineus group + Rhipicephalus bursa & 2 \\
Rhipicephalus sanguineus group + Ixodes canisuga & 1 \\
Rhipicephalus sanguineus group + Dermacentor reticulatus & 1 \\
Total & 24 \\
\hline
\end{tabular}




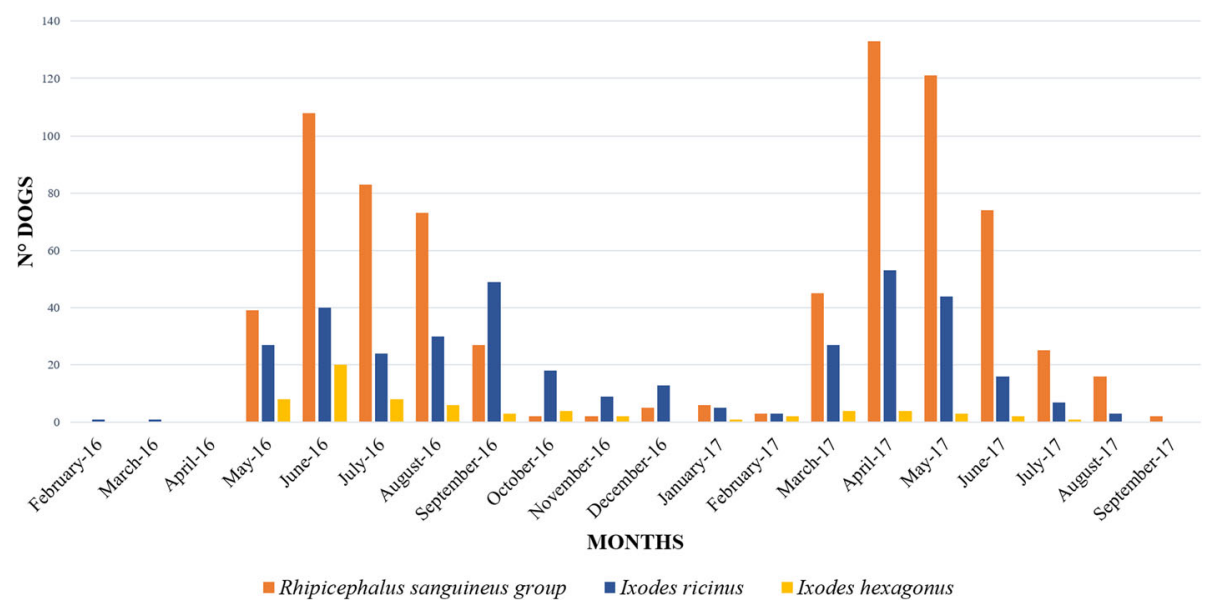

Fig. 4 Monthly seasonal dynamics of the three most prevalent ticks (Rhipicephalus sanguineus group, Ixodes ricinus and I. hexagonus) found on owned dogs in a survey in Italy (2016-2017)

interval for each product and the treatment indications against ticks of the genera Ixodes, Rhipicephalus and Dermacentor, oral formulations provided the highest protection, with $90.1 \%$ of dogs tick-free (95\% CI: 84.91-93.65\%) compared to $69.18 \%$ (95\% CI: $61.28-76.10 \%$ ) of dogs tick-free after treatment with antiparasitic collars (oral $v s$ collar: $\chi^{2}=$ 22.87, $d f=1, P<0.0001$ ) and 53.37\% (95\% CI: 48.94$57.75 \%$ ) of dogs were tick-free after treatment with a spot-on acaricide formulation (oral vs spot-on $\chi^{2}=77.08, d f$ $=1, P<0.0001)$. A significant higher efficacy was recorded for spot-on formulations compared to collars $\left(\chi^{2}=11.46, d f\right.$ $=1, P<0.001)$. Thirty-two different commercial products were used on dogs enrolled in the study for a total of 13 different active compounds (or active compound combinations). Only 5 compounds were used on more than 50 dogs with details provided of tick infestation prevalence related to compounds most frequently used on enrolled dogs (Table 5). No significant differences were recorded between compounds by generalized linear model (GLM) $(P>0.05)$; however, the greatest proportion of treated dogs that were tick free was with fluralaner (89.5\%). Owner respect for recommended retreatment intervals was greater for collars (95.4\% active collars), than for oral (70.8\% valid treatments) or spot-on formulations $(52.6 \%$ valid treatments). However, it was also apparent that dog owners and veterinarians lacked knowledge on acaricidal products because 31 dogs were reported to have been treated with products that have no acaricidal activity.

\section{Discussion}

To our knowledge, this is the first survey on ixodid tick prevalence and distribution on privately owned dogs across an Italian north-south and west-east transect. This study showed that a national tick prevalence survey can be conducted using voluntary practitioner enrolment, as previously carried out in other countries including Belgium [10], Spain [9] and the UK [12].

The geographical distribution of the four genera of ticks found across Italy in this survey is consistent with climatic and environmental features of the Italian peninsula and with maps of tick distribution in Europe (see maps in [26, 27]). Rhipicephalus species are widespread in the Mediterranean area while Ixodes spp. and Dermancentor spp. are better adapted to cold temperate and/or cold continental climates. The presence of Haemaphysalis (H. punctata) on dogs living outdoor in rural/sylvatic environments in southern Italy is consistent with this tick as the primary species infesting domestic ruminants in this area $[28,29]$. However, identification of 14 tick species confirms the high ixodid diversity [30] in Italy. Overall, $R$. sanguineus group (63.6\%) was the most prevalent, with high prevalence especially in the central-southern regions (36.1\%). This spatial distribution is

Table 5 Ectoparasiticide compounds used on at least 50 dogs in a survey in Italy, showing the proportion of dogs with ticks

\begin{tabular}{lllll}
\hline Compound & Dogs without ticks & Dogs with ticks & Total no. of dogs & Infestation prevalence (\%) \\
\hline Deltamethrin & 39 & 25 & 64 & 39.06 \\
Fipronil & 117 & 133 & 250 & 53.20 \\
Flumethrin - Imidacloprid & 61 & 18 & 79 & 22.78 \\
Fluralaner & 137 & 16 & 153 & 10.46 \\
Permethrin - Imidacloprid & 69 & 55 & 124 & 44.35 \\
\hline
\end{tabular}


associated with the suitable microclimate of the south for the development and maintenance of Rhipicephalus spp. [31]. Rhipicephalus sanguineus (s.l.) is the most prevalent species and this is a concern because it is the vector for various TBDs, including ehrlichiosis, anaplasmosis, and babesiosis. In southern Italy, other species belonging to the genus Rhipicephalus were found with much lower prevalence (less than 1\%), including $R$. bursa, $R$. pusillus, $R$. turanicus and Rhipicephalus sp. I. R. bursa and $R$. turanicus were reported in dogs from Basilicata, Campania, Latium, Sicily and Sardinia, regions where these species have been reported in livestock [24, 28, 29, 32-34] and where there is small ruminant farming, as recently hypothesized in Greece [35].

Ixodes ricinus was the second most common tick species in Italy (29.2\%). Higher prevalence values were recorded in the northern regions (20.9\%), but this tick was also found in central-southern Italy with a prevalence of 9.5\%. I. ricinus is a known vector for Lyme borreliosis and so the prevalence of this tick indicates the potential for this disease across Italy.

After $I$. ricinus, the five most prevalent other species of Ixodes were found: I. hexagonus (4\%), I. arboricola, I. canisuga and I. festai (prevalence below 1\%) in northern Italy, and I. hexagonus (1.2\%) and I. gibbosus (0.2\%) with low prevalence in southern regions.

The general spatial distribution of ticks in Italy observed in the present survey is consistent with findings of large-scale studies conducted in Mediterranean countries including Spain [9], Cyprus [13] and Greece [35]. These studies found $R$. sanguineus group to be the most prevalent tick species, while studies in temperate European countries including the Netherlands [36], Belgium [10] and UK [12] reported a higher prevalence of $I$. ricinus and I. hexagonus.

Further studies are needed to clarify ecological preferences for each detected tick species with respect to varying environmental and climatic features of the Italian peninsula and host availability.

The high prevalence of tick-infested dogs (45.7\%) in the present study should be carefully interpreted due to a possible over-reporting bias by practitioners as previously hypothesized in a large-scale UK survey [12]. Mixed infestation with two different tick species was found in 24 dogs and is thus quite rare as also reported in Spain [9] and in Greece [35]. In this study, a high number of ticks were adults as observed in other studies $[12,35]$ which could be a result of difficulties in detecting smaller tick life-stages (larvae and nymphs) during clinical examination [12].

Most ticks were attached to the head, the neck and the thorax/abdomen areas of the dog, and these are more exposed sites for tick attachment as previously reported [10, 37]. The difficulty for dogs to groom ticks from these areas, as well as the skin thickness and local odors could explain this multifocal distribution [31, 37]. Most ticks found in interdigital spaces were $R$. sanguineus, confirming this attachment site as a favorite for this species $[38,39]$. This result highlights the importance of systemic acaricidal treatments compared to topical ones because it is unlikely that topically applied and externally acting products will consistently reach acaricidal concentrations on distal locations such as the foot.

Results of our study showed that hair length and lifestyle (indoor $v s$ outdoor; urban $v s$ rural) were significant tick infestation predictors. A higher number of tick-infested dogs had long hair, possibly because of the greater difficulty for the owner to see and collect the ticks but also because long hair is easier for questing ticks to grab. This is consistent with one previous study [39] but is in contrast with another study [40] that found short-haired dogs significantly more likely to be in the highest infestation category compared with long-haired dogs. Finally, dogs living in rural/sylvatic environments or outdoor were more often tick-infested than pets living in urban areas or indoors as was also recently reported in Greece [35], although R. sanguineus (sensu lato) was the main group in both countries. No effect of age and sex was found on tick infestation. Previous reports also found that tick presence strongly correlated with tick exposure rather than any specific dog characteristic $[12,40]$. Overall, our analysis of the association between tick prevalence and dog characteristics further confirms the inconsistency of this relationship seen among prior tick risk surveys [12].

Seasonal tick species distribution in Italy showed considerable variation between genera. Rhipicephalus sanguineus group was most predominant during the spring and summer with an activity peak between April and August. In contrast, $I$. ricinus and $I$. hexagonus were found during all seasons, as observed in other European countries [9, 41-44].

The tick infestation risks for dogs in Italy observed in this study illustrate the importance of years round effective acaricidal treatment. Many owners are already treating their dogs with ectoparasiticidal compounds of a wide variety and types of formulation. Orally administered formulations showed the greatest efficacy; however, no currently available acaricidal treatment can completely prevent tick infestation and tick-borne diseases transmission. In this study, Rhipicephalus ticks were found to prefer the interdigital spaces, a difficult location for topical treatments to reach. This is consistent with the suggestion that systemic acaricidal treatment may offer better tick protection than externally distributed treatments. An additional possibility may be to consider combination therapy with both systemic and topically distributed actives [45]. 
The European Scientific Counsel for Companion Animal Parasites (ESCCAP) in Italy provides quality online information and advice on tick prevalence, tick treatments and tick borne pathogen risk (http://www.esccap.it). Veterinarians and pet owners should make more use of this information, for example to address gaps in their ectoparasiticidal product knowledge (which products are effective against ticks, and which are not). As seen in the results of this survey, several responses indicated that dogs at risk of tick infestation were treated with compounds that did not have an acaricidal effect. This may be due to the fact that most of the ectoparasiticidal products are sold "over the counter" facilitating the availability by owner but at the same time leading to an improper use of the drug.

The importance of using an effective treatment needs to be stressed, and communications from the ESCCAP website, including the parasite control guidelines, provide a reliable tick control information resource.

\section{Conclusions}

This first nationwide survey of ixodid ticks on companion animals in Italy has provided a comprehensive spatial understanding of tick distribution and species abundance, showing that different tick species parasitize dogs in this country. Risk factors vary by dog phenotype and lifestyle. Moreover seasonal species distribution showed considerable variation between tick genera. Further investigations are required to clarify the environmental and host factors that influence tick species infestations on companion animals, in order to develop and plan effective control measures.

\section{Abbreviations \\ DF: Degrees of freedom; ESCCAP: European Scientific Counsel for Companion Animal Parasites; EURNEGVEC: European Network for Neglected Vectors and Vector-Borne Infections; GLM: Generalized linear model; TBD: Tick-borne disease}

\section{Acknowledgements \\ The authors sincerely thank the COST Action TD1303 EURNEGVEC (European Network for Neglected Vectors and Vector-Borne Infections). The authors also acknowledge Professor Agustin Estrada-Peña, University of Zaragoza, Faculty of Veterinary Medicine, Spain, for his invaluable support in the identification of some submitted tick specimens. They would also thank all the veterinary practices who participated in this study.}

\section{Funding}

MSD Animal Health contributed to consumables costs, and Dr Liliana Colombo (Sr. Spclst. Scientific Marketing Affairs, MSD) handled the logistics of enrolling and registering veterinary practices. The MSD Animal Health sales team helped to recruit participating veterinary practices. Veterinarians did not receive payment for their participation.

\section{Availability of data and materials}

The data supporting the conclusions of this article are provided within the article.

\section{Authors' contributions}

MPM, PP and SZ supervised the data collection, undertook statistical analysis and drafted the manuscript. LC assisted in the design and management of the study and interpretation of data. RA reviewed the manuscript. LR, GC and EF conceived the study, coordinated and supervised the labor force and critically reviewed the manuscript. MPM, PP, EB, MEM, DC and SZ identified the ticks and carried out the molecular genetic studies and helped to draft the manuscript. All authors read and approved the final manuscript.

\section{Ethics approval}

This work was carried out with the approval of the University of Naples Federico II ethics committee.

\section{Consent for publication}

Not applicable.

\section{Competing interests}

The authors declare that they have no competing interests.

\section{Publisher's Note}

Springer Nature remains neutral with regard to jurisdictional claims in published maps and institutional affiliations.

\section{Author details}

${ }^{1}$ Department of Veterinary Medicine and Animal Production, University of Naples Federico II, Naples, Italy. ${ }^{2}$ MSD Animal Health, Milan, Italy. ${ }^{3}$ MSD Animal Health, Madison, NJ, USA. ${ }^{4}$ Department of Veterinary Sciences, University of Turin, Turin, Italy.

Received: 26 March 2018 Accepted: 5 July 2018

Published online: 16 July 2018

\section{References}

1. Animalplanetsthemostextremewiki. Bloodsuckers 2013. http:// animal planetsthemostextreme.wikia.com/wiki/Bloodsuckers. Accessed 01 Mar 2018.

2. Dantas-Torres F, Otranto D. Best practices for preventing vector-borne diseases in dogs and humans. Trends Parasitol. 2016:32:43-55.

3. Otranto D, Dantas-Torres F, Giannelli A, Latrofa MS, Cascio A, Cazzin S, et al. Ticks infesting humans in Italy and associated pathogens. Parasit Vectors. 2014;7:328.

4. Estrada-Peña A. Ticks as vectors: taxonomy, biology and ecology. Rev Sci Tech. 2015;34:53-65

5. Beugnet F, Marié JL. Emerging arthropod-borne diseases of companion animals in Europe. Vet Parasitol. 2009;163:298-305.

6. Smith FD, Ballantyne R, Morgan ER, Wall R. Prevalence, distribution and risk associated with tick infestation of dogs in Great Britain. Med Vet Entomol. 2011;25:377-84

7. Otranto D. Arthropod-borne pathogens of dogs and cats: from pathways and times of transmission to disease control. Vet Parasitol. 2018;251:68-77.

8. Aktas M. A survey of ixodid tick species and molecular identification of tickborne pathogens. Vet Parasitol. 2014;200:276-83.

9. Estrada-Peña A, Roura X, Sainz A, Miró G, Solano-Gallego L. Species of ticks and carried pathogens in owned dogs in Spain: results of a one-year national survey. Ticks Tick Borne Dis. 2017:8:443-52.

10. Claerebout E, Losson B, Cochez C, Casaert S, Dalemans AC, De Cat A, et al. Ticks and associated pathogens collected from dogs and cats in Belgium. Parasit Vectors. 2013:6:183.

11. Davies S, Abdullah S, Helps C, Tasker S, Newbury H, Wall R. Prevalence of ticks and tick-borne pathogens: Babesia and Borrelia species in ticks infesting cats of Great Britain. Vet Parasitol. 2017;244:129-35.

12. Abdullah S, Helps C, Tasker S, Newbury H, Wall R. Ticks infesting domestic dogs in the UK: a large-scale surveillance programme. Parasit Vectors. 2016;9:391.

13. Tsatsaris A, Chochlakis D, Papadopoulos B, Petsa A, Georgalis L, Angelakis E, et al. Species composition, distribution, ecological preference and host association of ticks in Cyprus. Exp Appl Acarol. 2016;70:523-42.

14. Dantas-Torres F, Maia C, Latrofa MS, Annoscia G, Cardoso L, Otranto D. Genetic characterization of Rhipicephalus sanguineus (sensu lato) ticks from dogs in Portugal. Parasit Vectors. 2017;10:133.

15. Morganti G, Gavaudan S, Canonico C, Ravagnan S, Olivieri E, Diaferia M, et al. Molecular survey on Rickettsia spp., Anaplasma phagocytophilum, Borrelia burgdorferi sensu lato, and Babesia spp. in Ixodes ricinus ticks infesting dogs in central Italy. Vector Borne Zoonotic Dis. 2017:17:743-8.

16. Satta G, Chisu V, Cabras P, Fois F, Masala G. Pathogens and symbionts in ticks: a survey on tick species distribution and presence of tick-transmitted microorganisms in Sardinia, Italy. J Med Microbiol. 2011;60:63-8. 
17. Corrain R, Drigo M, Fenati M, Menandro ML, Mondin A, Pasotto D, et al. Study on ticks and tick-borne zoonoses in public parks in Italy. Zoonoses Public Health. 2012:59:468-76.

18. Aureli S, Galuppi R, Ostanello F, Foley JE, Bonoli C, Rejmanek D, et al. Abundance of questing ticks and molecular evidence for pathogens in ticks in three parks of Emilia-Romagna region of northern Italy. Ann Agric Environ Med. 2015;22:459-66.

19. Dantas-Torres F, Otranto D. Species diversity and abundance of ticks in three habitats in southern Italy. Ticks Tick Borne Dis. 2013;4(3):251-5.

20. Di Luca M, Toma L, Bianchi R, Quarchioni E, Marini L, Mancini F, et al. Seasonal dynamics of tick species in an urban park of Rome. Ticks Tick Borne Dis. 2013:4:513-7.

21. Data bank of pet animals. Italian Ministry of Health. 2013. http://www.salute. gov.it/anagcaninapublic_new/home.jsp Accessed 21 May 2018

22. Walker A. Ticks of domestic animals in Africa. Edinburgh: Bioscience Reports; 2003. p. 3-210.

23. Estrada-Pena A, Bouattour A, Camicas JL, Walker AR. Ticks of domestic animals in the Mediterranean region. A guide to the identification of species. 1st ed. Zaragoza: University of Zaragoza; 2004.

24. Dantas-Torres F, Latrofa MS, Annoscia G, Giannelli A, Parisi A, Otranto D. Morphological and genetic diversity of Rhipicephalus sanguineus sensu lato from the New and Old Worlds. Parasit Vectors. 2013;6:213.

25. d'Oliveira $C$, van der Weide $M$, Jacquiet $P$, Jongejan $F$. Detection of Theileria annulata by the PCR in ticks collected from cattle in Mauritania. Exp Appl Acarol. 1997;21:279-91.

26. ESCCAP (European Scientific Counsel Companion Animal Parasites). ESCCAP Guideline 5 - Control of vector-borne diseases in dogs and cats. 2nd ed; 2012. http://www.esccap.org/uploads/docs/ih38c2d6_ESCCAP_Guidelines_ GL5_01Oct2012.pdf. Accessed 17 Mar 2017

27. Beugnet F, Halos L, Guillot J. Textbook of clinical parasitology in dogs and cats. Zaragoza: Merial; 2017.

28. Cringoli G, Otranto D, Testini G, Buono V, Di Giulio G, Traversa D, et al. Epidemiology of bovine tick-borne diseases in southern Italy. Vet Res. 2002; 33:421-8.

29. Rinaldi L, Otranto D, Veneziano V, Milillo P, Buono V, lori A, et al. Crosssectional survey of ticks (Acari: Ixodidae) in sheep from an area of the southern Italian Apennines. Exp Appl Acarol. 2004;33:145-51.

30. Manilla G. Fauna d'Italia. Acari: Ixodida. Calderini: Bologna; 1998

31. Lorusso V, Dantas-Torres F, Lia RP, Tarallo VD, Mencke N, Capelli G, et al. Seasonal dynamics of the brown dog tick, Rhipicephalus sanguineus, on a confined dog population in Italy. Med Vet Entomol. 2010;24:309-15.

32. Torina A, Khoury C, Caracappa S, Maroli M. Ticks infesting livestock on farms in western Sicily, Italy. Exp Appl Acarol. 2006;38:75-86.

33. Chisu V, Masala G, Foxi C, Socolovschi C, Raoult D, Parola P. Rickettsia conori israelensis in Rhipicephalus sanguineus ticks, Sardinia, Italy. Ticks Tick Borne Dis. 2014;5:446-8.

34. Toma L, Di Luca M, Mancini F, Severini F, Mariano C, Nicolai G, et al. Molecular characterization of Babesia and Theileria species in ticks collected in the outskirt of Monte Romano, Lazio Region. Central Italy. Ann Ist Super Sanita. 2017:53:30-4.

35. Latrofa MS, Angelou A, Giannelli A, Annoscia G, Ravagnan S, Dantas-Torres $F$, et al. Ticks and associated pathogens in dogs from Greece. Parasit Vectors. 2017;10:301.

36. Nijhof AM, Bodaan C, Postigo M, Nieuwenhuijs H, Opsteegh M, Franssen L, et al. Ticks and associated pathogens collected from domestic animals in the Netherlands. Vector Borne Zoonotic Dis. 2007;7:585-95.

37. Brianti E, Falsone L, Napoli E, Prudente C, Gaglio G, Giannetto S. Efficacy of a combination of $10 \%$ imidacloprid and $4.5 \%$ flumethrin (Seresto ${ }^{\odot}$ ) in slow release collars to control ticks and fleas in highly infested dog communities. Parasit Vectors. 2013;6:210.

38. Dantas-Torres F. Biology and ecology of the brown dog tick, Rhipicephalus sanguineus. Parasit Vectors. 2010;3:26.

39. Silveira JA, Passos LM, Ribeiro MF. Population dynamics of Rhipicephalus sanguineus (Latrielle, 1806) in Belo Horizonte, Minas Gerais state, Brazil. Vet Parasitol. 2009;161:270-5.

40. Jennett AL, Smith FD, Wall R. Tick infestation risk for dogs in a peri-urban park. Parasit Vectors. 2013;6:358

41. Duscher GG, Feiler A, Leschnik M, Joachim A. Seasonal and spatial distribution of ixodid tick species feeding on naturally infested dogs from eastern Austria and the influence of acaricides/repellents on these parameters. Parasit Vectors. 2013;6:76.
42. Földvári G, Farkas R. Ixodid tick species attaching to dogs in Hungary. Vet Parasitol. 2005;129:125-31.

43. Széll Z, Sréter-Lancz Z, Márialigeti K, Sréter T. Temporal distribution of Ixodes ricinus, Dermacentor reticulatus and Haemaphysalis concinna in Hungary. Vet Parasitol. 2006;141:377-9.

44. Dantas-Torres F, Otranto D. Seasonal dynamics of Ixodes ricinus on ground level and higher vegetation in a preserved wooded area in southern Europe. Vet Parasitol. 2013;192:253-8.

45. Pfister K, Armstrong R. Systemically and cutaneously distributed ectoparasiticides: a review of the efficacy against ticks and fleas on dogs. Parasit Vectors. 2016;9:436.

\section{Ready to submit your research? Choose BMC and benefit from:}

- fast, convenient online submission

- thorough peer review by experienced researchers in your field

- rapid publication on acceptance

- support for research data, including large and complex data types

- gold Open Access which fosters wider collaboration and increased citations

- maximum visibility for your research: over $100 \mathrm{M}$ website views per year

At BMC, research is always in progress.

Learn more biomedcentral.com/submissions 